\title{
The comparison and diagnostic accuracy of different types of thermometers
}

\author{
Nurettin Erdem ${ }^{1 \oplus}$, Tuğba Bedir Demirdağ ${ }^{2 \oplus}$, Hasan Tezer $^{2 \oplus}$, \\ Burcu Ceylan Cura Yayla ${ }^{2 \oplus}$, Fatma Nur Baran Aksakal ${ }^{3 \oplus}$, Anil Tapisiz $^{2 \oplus}$, \\ Okşan Derinöz ${ }^{4 \oplus}$, Arzu Okur ${ }^{5 \oplus}$, Faruk Güçlü Pınarl1 ${ }^{5 \oplus}$, Ülker Koçak ${ }^{5 \oplus,}$ \\ Aysun Bideci ${ }^{6 \oplus}$ \\ Departments of ${ }^{1}$ Pediatrics, ${ }^{2}$ Pediatic Infectious Diseases, ${ }^{3}$ Public Health, ${ }^{4}$ Pediatric Emergency, ${ }^{5}$ Pediatric Hematology and Oncology, \\ and ${ }^{6}$ Pediatric Endocrinology Gazi University Faculty of Medicine, Ankara, Turkey.
}

\begin{abstract}
Background. Fever is one of the leading causes of hospital admissions in children. Although there are many ways to measure body temperature, the optimal method and the anatomic site are still controversial. In this study, we aimed to evaluate the performance of new methods of measuring body temperature and to compare the accuracy, sensitivity and specificity of these methods.
\end{abstract}

Methods. The body temperatures of the patients who were hospitalized as inpatients or who presented to the emergency room as outpatients between November 2014- March 2015 were measured and recorded. Mercury and digital axillary measurements, tympanic, temporal artery and non-contact skin temperatures were measured. Measurements were compared with each other.

Results. According to our results temperature tends to increase over time for up to 8 minutes after placement when using axillary thermometers. Non-contact skin thermometers should be used only for follow-up of patients with fever, because of their low sensitivity and low negative predictivity. At the first examination, tympanic thermometers and axillary thermometers may be preferable for the diagnosis of fever.

Conclusions. According to our results, using non-contact thermometers seems feasible and logical during the follow-up ofpatients with fever, but not in cases whose exact body temperature should be known. For the first examination of the patient to diagnose fever, tympanic thermometers and axillary thermometers may be preferable. Future studies are warranted to expose the optimum way of measuring body temperature in children.

Key words: body temperature, thermometers, pediatrics.

Fever is one of the leading causes of hospital admissions for children. ${ }^{1}$ Although it is an important "self-defense" mechanism, it often triggers significant fear and anxiety among caregivers. $^{2}$

The purpose of body temperature monitoring is to approximate the core temperature and the temperature of the pulmonary artery, as

$凶$ Tuğba Bedir Demirdağ

tugbabedir@gmail.com

Received 16th October 2019, revised 26th August 2020, accepted 12th September 2020. accurately as possible. ${ }^{3}$ Rectal measurement is practically accepted as the core temperature. ${ }^{1}$ Although rectal measurement is known to be the gold standard method of measuring body temperature; it may cause traumatic or infectious complications. ${ }^{1,4}$

Tympanic and non-contact measurements of body-temperature from the skin are new alternatives to mercury thermometers. Although there are many advantages and disadvantages to each method of measuring body temperature, the selection of the appropriate method and anatomic site still remains controversial. An 
ideal method should make measurements as close as possible to core temperature rapidly and accurately across all age groups, should minimize cross-contamination, and should have an excellent safety profile. Cost and ease of calibration are also important concerns. ${ }^{1}$

Herein we aimed to evaluate the performance of new methods for measuring body temperature and to compare the sensitivity, specificity, and accuracy of these methods. For this purpose, we applied axillary mercury thermometers, axillary digital thermometers, tympanic thermometers, temporal artery thermometers and noncontact skin thermometers for measuring body temperature to our patient cohort and compared the results obtained using each.

\section{Material and Methods}

Body temperatures of all pediatric patients who were admitted as inpatients or applied to the emergency room as outpatients were recorded between the dates of November 1, 2014 and February 28, 2015. Informed consent was provided by the parents of all patients.

Patient demographics, social status, and physical examination findings were recorded for each patient. The final study group consisted of a total of 151 patients and 2265 body temperature measurements were collected (15 times for each patient).

Fever was defined as a rectal temperature $\geq 38^{\circ} \mathrm{C} .{ }^{5}$ But rectal measurement was not used in this study and body temperature $\geq 38^{\circ} \mathrm{C}$ was accepted as fever for all methods.

The thermometer accuracy was checked using a $38^{\circ} \mathrm{C}$ water bath before each measurement.

Thermometers were calibrated according to the product manual. The measurements were taken at $24^{\circ} \mathrm{C}$ room temperature $\left(24^{\circ} \mathrm{C}\right)$, allowing 10 minutes for the patient to become acclimated to ambient temperature before the measurement.

Body temperatures were measured by axillary mercury thermometers, axillary digital thermometers, tympanic thermometers, temporal artery thermometers and non-contact skin thermometers for each patient. The axilla and forehead were dried before the measurement with non-contact thermometers. A tympanic thermometer was placed in the outer third of the external auditory canal. The tragus was pulled down and back in children aged less than three years, and up and back in children aged greater than three years. After placement of the thermometer, a signal from the thermometer indicated completion of the measurement. Non-contact skin thermometers were also used according to the instructions and body temperature was measured from the forehead. Similarly, body temperature was measured with temporal artery thermometers(TAT) according to the instructions from the manufacturer. Temperatures of the skin over the temporal artery, on the temple, and on the mastoid process were measured. Mercury and digital thermometers were placed at the axilla and for eight minutes. The temperatures were recorded at the end of the 3rd, 5th, and 8th minute. For each kind of thermometer, these measurements were repeated three times with a total number of 15 measurements for each patient.

All statistical analyses were carried out using IBM SPSS Statistics, version 20(SPSS Inc, Chicago, IL, USA). Homogeneity of the distribution of variables was evaluated using the chi-square test. Non-parametric tests were used in cases of non-normal variable distribution. Comparison of the methods was done using the Spearman correlation test and Bland-Altman test.

The study protocol was approved by the ethics committee of Gazi University, with the reference number 2014/112.

\section{Results}

One hundred and fifty-one patients were included in the study. A total of 2,265 body temperature measurements were collected (15 times for each patient). Eighty-one of the patients were male (54\%) and 70 patients were 
female $(46 \%)$. The male/female ratio was 1.15. There was no significant difference in gender distribution $(\mathrm{P}=0.374)$.

The age range of the study group was 2-18 years. The mean age was $7.95 \pm 4.48$ years. The median age was 7.4 (2.5-17) years. The distribution of patients by age and sex was homogeneous.

While 83 patients were hospitalized, 68 patients were admitted to the emergency service as outpatients.

The clinical diagnoses of the patients were as follows: upper respiratory infection $(n=83$, $55 \%)$, lower respiratory infection $(n=18$, $11.9 \%)$, gastrointestinal diseases $(\mathrm{n}=12,7.9 \%)$, haematologic diseases $(n=9,6 \%)$, urinary tract infections $(n=7,4.6 \%)$, oncologic diseases $(n=6$,
$4 \%)$, cellulitis $(n=5,3.3 \%)$, lymphadenitis $(n=5$, $3.3 \%)$ and nephrologic diseases $(n=5,3.3 \%)$.

The 3rd, 5th and 8th measurements of each method (mercury thermometer, digital axillary thermometer (AT), TAT, and non-contact skin thermometer) were compared individually, using the Friedman test. The 8th minute measurements of digital axillary and mercury thermometer measurements were significantly higher than 3rd and 5th minute measurements of these methods ( $\mathrm{p}=0.00$; Table I-II-III).

The values measured using a tympanic thermometer were tested using the Friedman test.

There was no significant difference between the measurement methods among patients more

Table I. Comparison of methods of body-temperature measurement.

\begin{tabular}{|c|c|c|c|c|}
\hline & & 3rd minute & 5th minute & 8th minute \\
\hline \multirow[t]{5}{*}{ Mercury Thermometer } & Mean & 37.52 & 37.67 & 37.81 \\
\hline & Median & 37.80 & 37.80 & 38.00 \\
\hline & Standart deviation & 1.16 & 1.17 & 1.20 \\
\hline & Min & 35.20 & 35.10 & 35.50 \\
\hline & Max & 40.00 & 40.30 & 40.60 \\
\hline \multirow[t]{5}{*}{ Digital Axillary Thermometer } & Mean & 37.50 & 37.61 & 37.71 \\
\hline & Median & 37.80 & 37.80 & 37.80 \\
\hline & Standart deviation & 1.24 & 1.26 & 1.30 \\
\hline & Min & 35.50 & 34.50 & 34.30 \\
\hline & Max & 40.10 & 40.30 & 40.30 \\
\hline \multirow[t]{5}{*}{ Temporal Artery Thermometer } & Mean & 38.2146 & 38.3007 & 38.3291 \\
\hline & Median & 37.90 & 37.90 & 38.10 \\
\hline & Standart deviation & 1.2148 & 1.2886 & 1.2790 \\
\hline & Min & 35.90 & 35.90 & 35.90 \\
\hline & Max & 41.80 & 41.70 & 42.10 \\
\hline \multirow[t]{5}{*}{ Non-contact Skin Thermometer } & Mean & 37.5026 & 37.5444 & 37.5603 \\
\hline & Median & 37.30 & 37.40 & 37.40 \\
\hline & Standart deviation & 1.0075 & 1.0153 & 1.0263 \\
\hline & Min & 36.00 & 36.10 & 35.90 \\
\hline & Max & 40.00 & 39.90 & 40.10 \\
\hline \multirow[t]{5}{*}{ Tympanic Thermometer } & Mean & 37.64 & 37.56 & 37.56 \\
\hline & Median & 37.70 & 37.60 & 37.70 \\
\hline & Standart deviation & 1.10 & 1.07 & 1.07 \\
\hline & Min & 35.20 & 35.10 & 35.10 \\
\hline & Max & 40.20 & 40.20 & 40.30 \\
\hline
\end{tabular}


Table II. Comparison of methods of body-temperature measurement.

\begin{tabular}{llllll}
\hline & Digital & Non-contact & Tympanic & Mercury & Temporal artery thermometer \\
\hline Mean & 37.71 & 37.56 & 37.56 & 37.81 & 38.32 \\
Median & 37.80 & 37.40 & 37.70 & 38.00 & 38.10 \\
SD & 1.30 & 1.02 & 1.07 & 1.20 & 1.27 \\
Min & 34.30 & 35.90 & 35.10 & 35.50 & 35.90 \\
Max & 40.30 & 40.10 & 40.30 & 40.60 & 42.10 \\
\hline
\end{tabular}

Table III. The comparison of mercury thermometer values with other methods.

\begin{tabular}{lllll}
\hline & Specifity & Senstivity & Positive predictivity & Negative predictivity \\
\hline Digital axillary thermometer & $98 \%$ & $85 \%$ & $98 \%$ & $92 \%$ \\
Temporal artery thermometer & $94 \%$ & $93 \%$ & $94 \%$ & $88 \%$ \\
Non-contact thermometer & $100 \%$ & $69 \%$ & $100 \%$ & $93 \%$ \\
Tympanic thermometer & $98 \%$ & $87 \%$ & $98 \%$ & $92 \%$ \\
\hline
\end{tabular}

than 12 years of age $(p=0.64)$. However, among patients under 12 years of age the difference betweenthe measurement methods was significant $(\mathrm{p}=0.04)$, (Table I).

The mean and the median 8th minute measurements of mercury, digital, noncontact skin,and TAT were compared. TAT measurements were significantly higher than othermethods (Table I).

Defining fever as an axillary temperature of $>38^{\circ} \mathrm{C}$ the specificity, sensitivity, positive and negative predictive values of each measurement technique were calculated (Table III).

The distribution of temperature measurements did not conform to a normal distribution and; therefore, the spearman correlation test was used. There was a strong positive correlation between the mean 8th minute measurementof the mercury thermometer and that of the tympanic thermometer (Fig 1), the non-contact skin thermometer (Fig. 2), the TAT (Fig. 3) and the digital AT (Fig. 4), (Sperman's rho: 0.77, $\mathrm{p}=0.001$ ) within a confidence interval of $95 \%$.

\section{Discussion}

The aim of this study was to compare body temperature measurement methods. To the best of our knowledge, this study includes one of the largest pediatric data. One of the most important points of this study is that temperature tends to increase over time for up to 8 minutes after placement when using axillary mercury and axillary digital thermometers. Secondly, according to our results non-contact skin thermometers should be used only for the follow-up of patients with fever, because of their low sensitivity and low negative predictivity.

The first method used to measure body temperature was an AT. The American Academy of Pediatrics recommends the use of ATs to measure body temperature, despite its relatively low sensitivity and specificity. ${ }^{1}$ In a review, Craig et al. ${ }^{4}$ concluded that axillary measurement might be inaccurate and was not ideal for body temperature measurement.

There is no consensus on the optimal duration for retaining ATs in the armpit. Common protocols vary from 1-15 minutes, but 4-7 minutes are generally accepted assufficient..$^{6-8}$ Chaturvedi et al. ${ }^{8}$ showed that temperature was stable after five minutes after the placement of the thermometer in $76 \%$ of patients. When we evaluated axillary measurements, the mean temperature at the 8 th minutes $\left(37.8^{\circ} \mathrm{C}\right)$ was greater than the means of the $3 \mathrm{rd}\left(37.5^{\circ} \mathrm{C}\right)$ and 5th minutes $\left(37.6^{\circ} \mathrm{C}\right)$. Erdal et al..$^{9}$ have claimed that mercury thermometer measurements stabilize at 4-6 minutes. Greylinget al. ${ }^{10}$ 


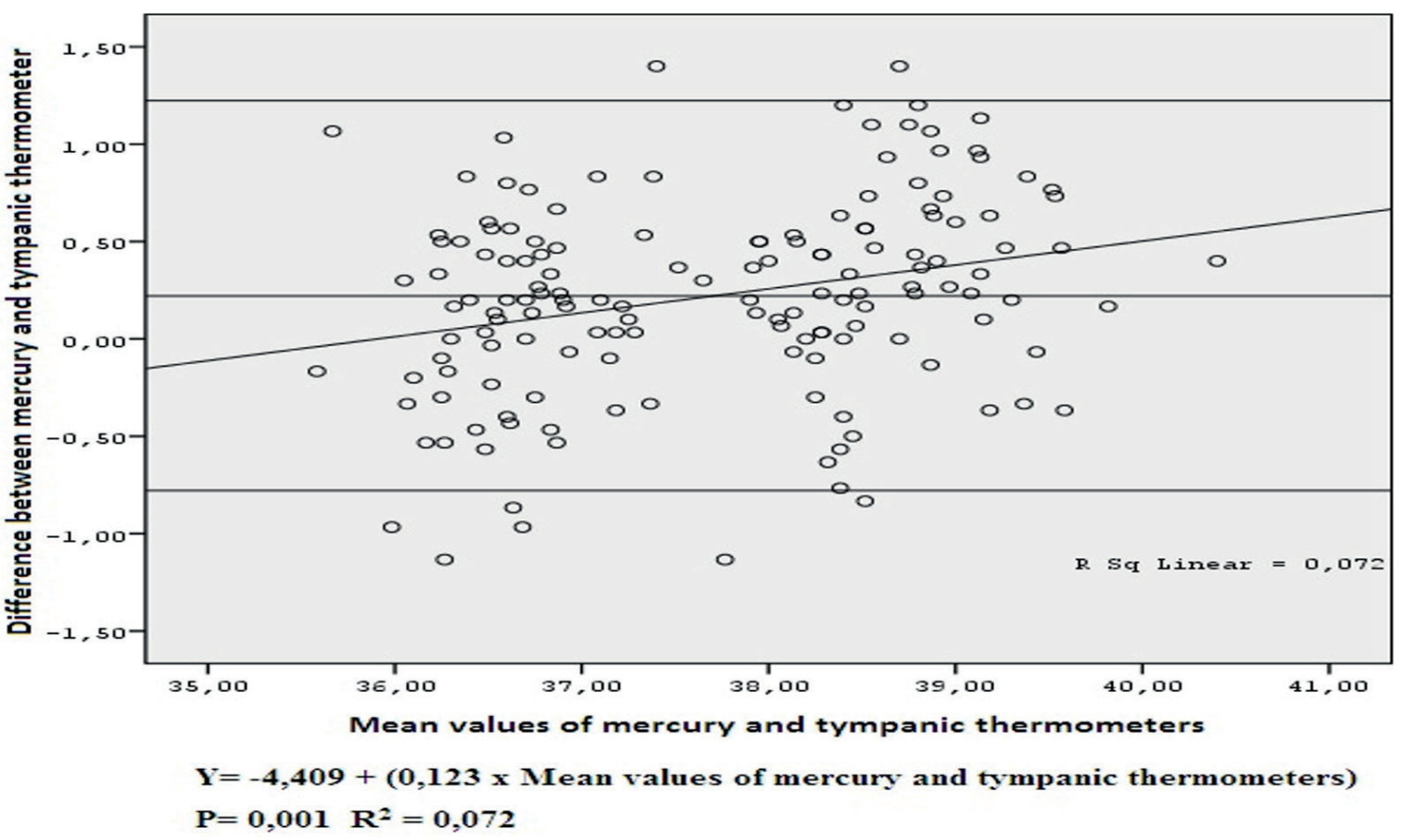

Fig. 1. Tympanic thermometer Bland altman test results.

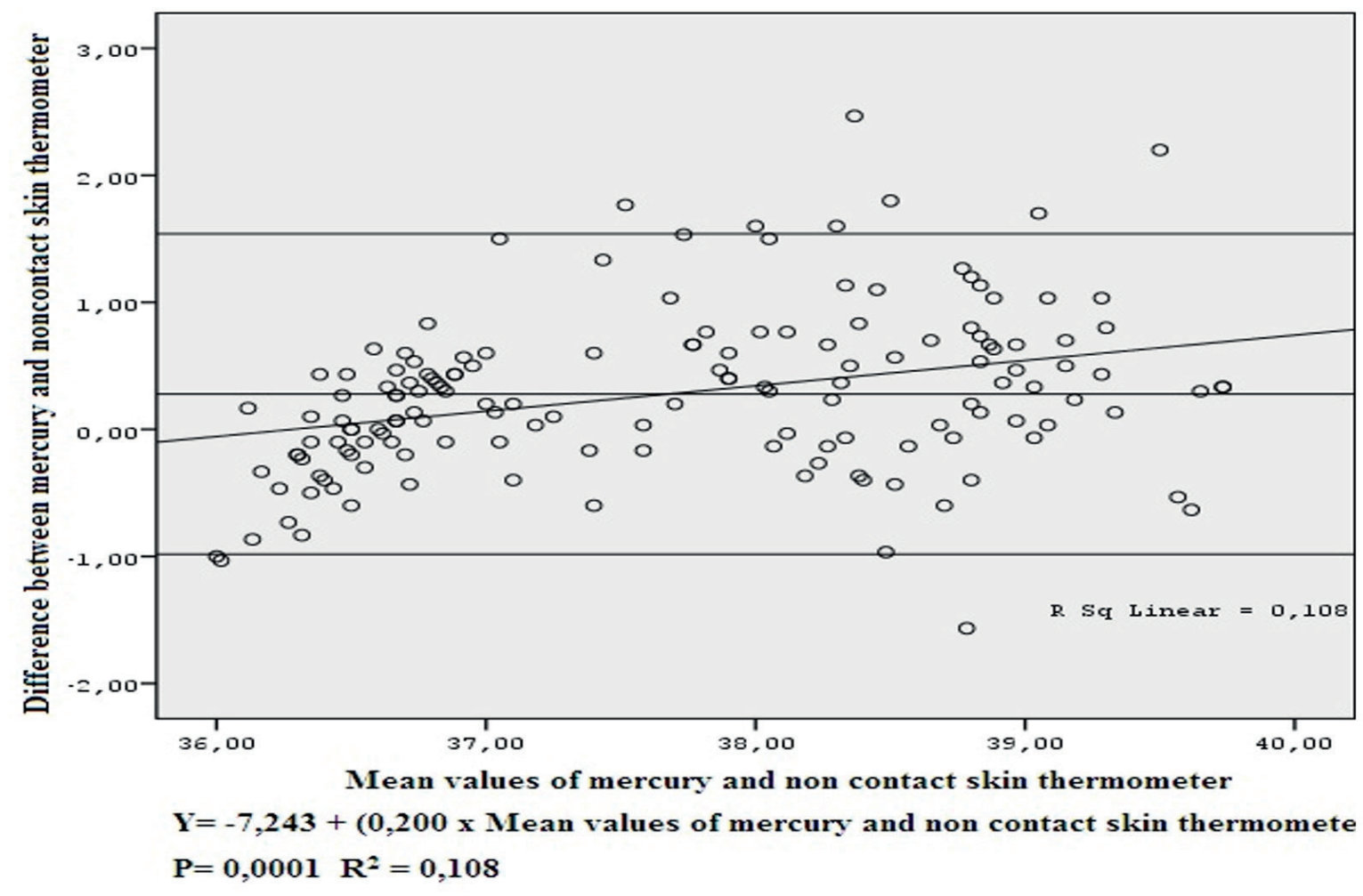

Fig. 2. Non-contact skin thermometer Bland altman test results. 


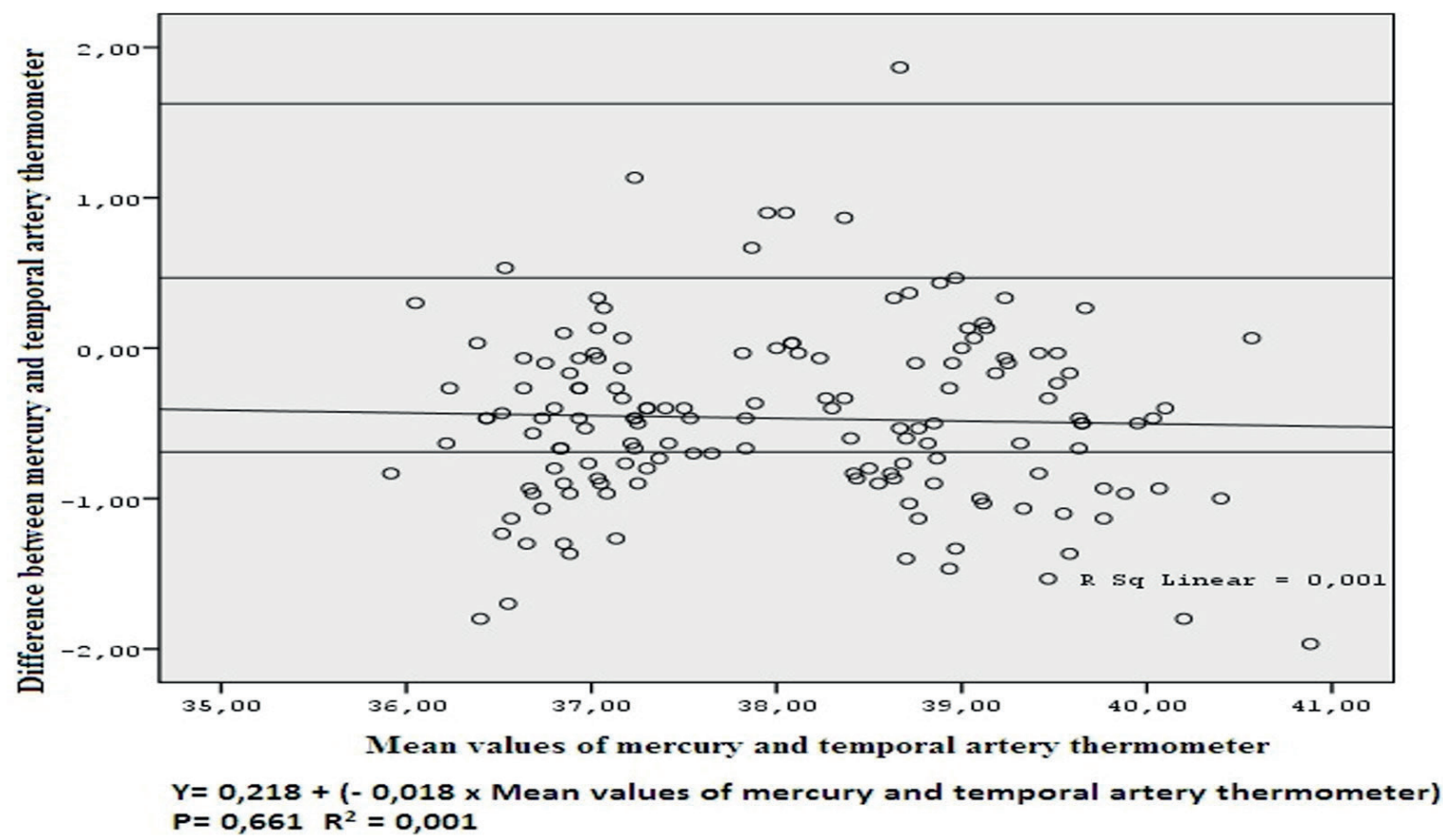

Fig. 3. Temporal artery thermometers Bland altman test results.

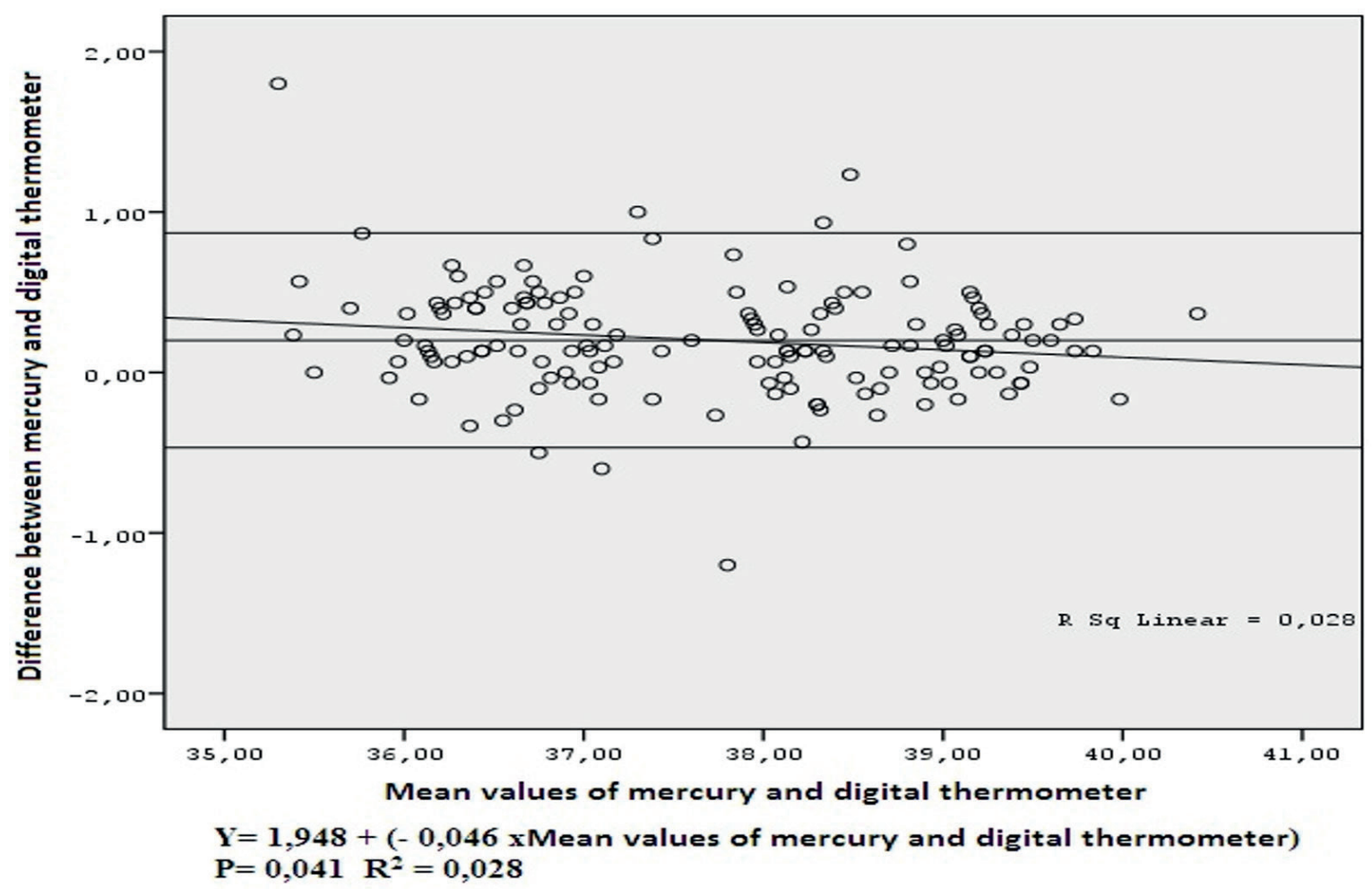

Fig. 4. Digital thermometer Bland altman test results. 
concluded that the $9^{\text {th }}$ minute value had the greatest sensitivity. Khordish et al. ${ }^{6}$ indicated that the increase in temperature after eight minutes was not statistically significant. Similar to the literature, we found that temperature tends to increase over time for up to the 8th minute after placement when using axillary mercury and digital thermometers.

In 2001 Latman et al. ${ }^{11}$ proposed that digital thermometers may be used as a replacement for mercury thermometers. According to the sensitivity and specificity of ATs, our study supports the use of digital thermometers in place of mercury thermometers. ${ }^{4}$

The TAT is a newer method for measuring body temperature ultra-red waves and measuring

the highest temperature on the temporal artery trace. Batra et al. ${ }^{12}$ established that temporal artery temperature is similar to rectal body temperature and is better than axillary or tympanic temperature at approximating core temperature. In 2014, Isler et al. ${ }^{13}$ stated that the TATs can be safely used instead of ATs. Besides positive aspects of TATs, there are some studies indicating that the sensitivity of temporal artery temperature measurement is low. Siberry and Hoffman ${ }^{14,15}$ claimed that TATs had low sensitivity. Greenes et al. ${ }^{16}$ showed that TATs were more reliable than ATs, but their sensitivity became lower at high temperatures. In our study, the difference between the temporal artery temperature and the axillary temperature was $0.7^{\circ} \mathrm{C}$ at 3 minutes and $0.5^{\circ} \mathrm{C}$ at 8 minutes. We found that the $3 \mathrm{rd}$ minute values were similar to reports in the literature, although 8th minute values differed from previous reports. We demonstrated that if the waiting period ( 8 minute) and technique were appropriate, ATs resulted in temperature measurements comparable to TATs.

Non-contact skin thermometers resulted in $0.7^{\circ} \mathrm{C}$ lower body temperature measurements when compared to ATs. In a study involving 179 newborns, Can et al. ${ }^{17}$ showed that non-contact thermometers measured body temperature $0.5^{\circ} \mathrm{C}$ higher than digital or mercury ATs. Our findings probably differ as a result of the older patient population that we examined. Batra and Goyal ${ }^{12}$ found that non-contact skin temperature was the closest approximation to rectal body temperature. Conversely, Fortuna et al. ${ }^{18}$ found that non-contact skin thermometers correlated poorly with rectal thermometers and should not be used routinely in pediatric patients. Paes etal. ${ }^{19}$ claimed that non-contact skin thermometers could be used when rectal thermometers are not feasible. We compared non-contact skin temperature with axillary temperature and we found a strong, positive and statistically significant correlation between the two measurement techniques. When mercury thermometer measurement $>38{ }^{\circ} \mathrm{C}$ is accepted as fever, non-contact skin thermometer measurements had 100\% specificity, 69\% sensitivity, $69 \%$ positive predictivity and $93 \%$ negative predictivity. Thus, we recommend that non-contact thermometers should be used only for the follow-up of patients with fever, because of their low sensitivity and low negative predictivity.

The tympanic membrane accurately reflects core temperature. Barton and Kocaoğlu ${ }^{20,21}$ determined that the tympanic measurement method had the best correlation with rectal measurement and that it was strongly preferred by patients. However, Lanham et al. ${ }^{22}$ showed that the tympanic measurement technique had very low sensitivity and specificity when compared to rectal measurement. According to our study, tympanic thermometers have a strong correlation with ATs and have acceptable sensitivity and specificity. However, the fact that recurrent measurements result in different values among the patients under 12 may raise doubts about the reliability of this method. This technique is more difficult in younger children resulting in reduced reliability. ${ }^{23}$ Tympanic thermometers may be preferred due to ease of use and correlation with rectal measurements, however practitioners should keep in mind that this method may not be the most accurate approach in young children. 
In conclusion, there is still no consensus concerning the best way of measuring body temperature and it is still an unclear issue in pediatrics. This study aimed to present the most appropriate method including the newer techniques. According to our results, using noncontact thermometers seems feasible and logical in the follow-up of the patients with fever, but not in cases whose exact body temperature should be known. Tympanic thermometers and axillary thermometers may be preferable for the initial diagnosis of fever. Future studies are warranted to expose the optimum way of measuring body temperature in children.

\section{Author contribution}

The contributions of all authors must be described in the following manner: The authors confirm contribution to the paper as follows: study conception and design: Hasan Tezer, Tuğba Bedir Demirdağ; data collection: Nurettin Erdem, Tuğba Bedir Demirdağ, Burcu Ceylan Cura Yayla, Anıl Tapısız, Arzu Okur, Okşan Derinöz, Faruk Güçlü Pınarlı, Ülker Koçak, Aysun Bideci; analysis and interpretation of results: Hasan Tezer, Fatma Nur Baran Aksakal; draft manuscript preparation: Tuğba Bedir Demirdağ, Nurettin Erdem. All authors reviewed the results and approved the final version of the manuscript.

\section{Ethical approval}

The study protocol was approved by the ethics committee of Gazi University, with the reference number 2014/112.

\section{Source of funding}

No funding resources.

\section{Conflict of interest}

The authors have no conflicts of interest relevant to this article to disclose.

\section{REFERENCES}

1. El-Radhi AS, Carroll J, Klein N. Clinical Manual of Fever in Children (1st ed) Berlin Heidelberg: Springer-Verlag, 2009.

2. MayA, Bauchner H.Fever phobia: the pediatrician's contribution. Pediatrics 1992; 90: 851-854.

3. Nierman DM. Core temperature measurement in the intensive care unit. CritCare Med 1991; 19: 818-823.

4. Craig JV, Lancaster GA, Williamson PR, Smyth RL. Temperature measured at the axilla compared with rectum in children and young people: systematic review. BMJ 2000; 320: 1174-1178.

5. Powell KR. Chapter 174: Fever. Kliegman RM, Behrman RE, Jenson HB, Stanton BF. Nelson Textbook of Pediatrics (18th ed) Philedelphia: Elsevier, 2007: 1084-1087.

6. Khorshid L, Eşer İ, Zaybak A, Yapucu U. Comparing mercury-in-glass, tympanic and disposable thermometers in measuring body temperature in healthy young people. J Clin Nurs 2005; 14: 496-500.

7. Gardner S. Axillary and rectal temperatures of full term neonates: are they different? Neonatal Netw 1987; 5: 42-43.

8. Chaturvedi D, Vilhekar KY, Chaturvedi P, Bharambe MS. Comparison of axillary temperature with rectal or oral temperature and determination of optimum placement time in children. Indian Pediatr 2004; 41: 600-603.

9. Erdal E, Eşer İ, Demir Ü ve ark.Oral ve aksiller yolla beden sıcaklığ1 ölçümünde termometrelerin bekletilme sürelerinin incelenmesi. Ege Üniversitesi Hemşirelik Yüksek Okulu Dergisi 1991; 7: 23-28.

10. Greyling G, Viljoen MJ, Joubert G. Axillary temperature compared to tympanic membrane temperature in children. Curationis 2000; 23: 54-61.

11. Latman NS, Hans P, Nicholson L, DeLee ZintS, Lewis $\mathrm{K}$, Shirey A. Evaluation of clinical thermometers for accuracy and reliability. Biomed Instrum Technol 2001; 35: 259-265.

12. Batra P, Goyal S. Comparison of rectal, axillary, tympanic, and temporal artery thermometry in the pediatric emergency room. Pediatric Emerg Care 2013; 29: 63-66.

13. Işler A, Aydin R, Tutar Güven Ş, Günay S. Comparison of temporal artery to mercury and digital temperature measurement in pediatrics. Int Emerg Nurs 2014; 22: 165-168.

14. Siberry GK, Diener-West M, Schappell E, Karron RA. Comparison of temple temperatures with rectal temperatures in children under two years of age. Clin Pediatr (Phila) 2002; 41: 405-414. 
15. Hoffman RJ, Etwaru K, Dreisinger N, Khokhar A, Husk G. Comparison of temporal artery thermometry and rectal thermometry in febrile pediatric emergency department patients. Pediatr Emerg Care 2013; 29: 301-304.

16. Greenes DS, Fleisher GR. Accuracy of a noninvasive temporal artery thermometer for use in infants. Arch Pediatr Adolesc Med 2001; 155: 376-381.

17. Can E, BülbülA, UsluS, NuhoğluA. Yenidoğan yoğun bakım birimindeki hastalarda temassız infrared alın termometresi ile standart termometrelerin karşılaştırılması. Türk Pediatri Arşivi Dergisi 2010; 45: 257-263.

18. Fortuna EL, Carney MM, Macy M, Stanley RM, Younger JG, Bradin SA. Accuracy of non-contact infrared thermometry versus rectal thermometry in young children evaluated in the emergency department for fever. J Emerg Nurs 2010; 36: 101104.

19. Paes BF, Vermeulen K, Brohet RM, van der Ploeg $\mathrm{T}$, de Winter JP. Accuracy of tympanic and infrared skin thermometers in children. Arch Dis Child 2010; 95: 974-978.
20. Barton SJ, Gaffney R, Chase T, Rayens MK, Piyabanditkul L. Pediatric temperature measurement and child/parent/nurse preference using three temperature measurement instruments. J Pediatr Nurs 2003; 18: 314-320.

21. Kocoglu H, Goksu S, Isik M, Akturk Z, Bayazit YA. Infrared tympanic thermometer can accurately measure the body temperature in children in an emergency room setting. Int $\mathrm{J}$ Pediatr Otorhinolaryngol 2002; 65: 39-43.

22. Lanham DM, Walker B, Klocke E, Jennings M. Accuracy of tympanic temperature readings in children under 6 years of age. Pediatr Nurs 1998; 25: 39-42.

23. El-Radhi A, Patel S. An evaluation of tympanic thermometry in a paediatric emergency department. Emerg Med J 2006; 23: 40-41. 